\title{
In Vivo Validation of a Bioinformatics Based Tool to Identify Reduced Replication Capacity in HIV-1
}

\author{
Christina M.R. Kitchen ${ }^{*}, 1,2$, Paul Krogstad ${ }^{2,3}$ and Scott G. Kitchen ${ }^{2,4}$ \\ ${ }^{I}$ Department of Biostatistics, UCLA School of Public Health, ${ }^{2}$ UCLA AIDS Institute, ${ }^{3}$ Departments of Pediatrics and \\ Molecular and Medical Pharmacology, and ${ }^{4}$ Division of Hematology/Oncology, Department of Medicine, The David \\ Geffen School of Medicine at UCLA, Los Angeles, California 90095, USA
}

\begin{abstract}
Although antiretroviral drug resistance is common in treated HIV infected individuals, it is not a consistent indicator of HIV morbidity and mortality. To the contrary, HIV resistance-associated mutations may lead to changes in viral fitness that are beneficial to infected individuals. Using a bioinformatics-based model to assess the effects of numerous drug resistance mutations, we determined that the D30N mutation in HIV-1 protease had the largest decrease in replication capacity among known protease resistance mutations. To test this in silico result in an in vivo environment, we constructed several drug-resistant mutant HIV-1 strains and compared their relative fitness utilizing the SCID-hu mouse model. We found HIV-1 containing the D30N mutation had a significant defect in vivo, showing impaired replication kinetics and a decreased ability to deplete CD4+ thymocytes, compared to the wild-type or virus without the D30N mutation. In comparison, virus containing the M184V mutation in reverse transcriptase, which shows decreased replication capacity in vitro, did not have an effect on viral fitness in vivo. Thus, in this study we have verified an in silico bioinformatics result with a biological assessment to identify a unique mutation in HIV-1 that has a significant fitness defect in vivo.
\end{abstract}

Keywords: HIV-1, replication capacity, bioinformatics, Bayesian, variable selection, exchangeable on subsets, prior model selection, in vivo validation.

\section{INTRODUCTION}

The rate of HIV-1 replication and mutation in infected individuals is remarkable and alarming [1-3]. During antiretroviral treatment there is a dynamic interplay of drugresistance and fitness occurring within the virus population. It has been proposed that reduced viral fitness of HIV-1 can result in slower disease progression in vivo due to the decreased pathogenic ability of the virus [4-6]. Viral fitness in this case is defined by the ability of a virus to replicate in vivo and produce pathologic changes in host tissues. While it is difficult to directly assess viral fitness in vivo, the relative replication capacity (RC) of a viral mutant may be determined using in vitro cell culture models by examining how the virus replicates when compared to a virus without any mutations (wild-type virus) [4, 7, 8]. Viral fitness in vivo and observed RC in vitro are clearly linked; however, there is a lack of direct evidence that certain drug-resistance mutations actually confer a defect in viral fitness in vivo in infected persons.

In the clinical setting, combination antiretroviral therapy (ART) often fails to completely and durably suppress plasma levels of HIV-1 RNA [9-12] and the viral load may rebound. Virologic failure is often a consequence of a series of mutations in HIV that decreases the susceptibility of the virus to antiretroviral agents. It has been shown that drugresistant HIV often has lower RC in vitro than wild-type

*Address correspondence to this author at the Department of Biostatistics, UCLA School of Public Health, BOX 951772, 21-257 CHS, Los Angeles, CA 90095-1772, USA; Tel: 310-825-7332; Fax: 310-267-2113;

E-mail: cr@ucla.edu virus $[4,7,8,13]$. Treatment interruption of ART in patients leads to the re-emergence of archived wild-type virus that has a higher $\mathrm{RC}$ than the circulating drug-resistant virus and is associated with higher viremia and decreased CD4 T-cell counts $[14,15]$. In contrast, patients who remain on their ART regimen despite ongoing viral replication may have stable CD4 counts and stable viremia $[5,16]$. In one report, only $36.8 \%$ of patients experienced a decrease in CD4 counts to pre-therapy levels while remaining on a failing ART regimen for 3 years [16]. Similarly, Barbour et al. [17] found patients who remained on a failing regimen had stable $\mathrm{RC}$, viremia and CD4 counts. These studies suggest that HIV is subject to genetic bottlenecks where it cannot create further resistance without sacrificing its ability to replicate.

Decreases in viral RC due to mutation have been wellcharacterized in vitro, although alterations in fitness in vivo is often correlative due to the lack of use of a controlled experimental system during these studies [4, 7, 8, 13, 17-20]. It has been shown that certain mutations cause a substantial decrease in $\mathrm{RC}$ in vitro relative to wild-type strains. However, there also exist mutants that have similar or even higher replication capacity than wild-type [21]. The D30N mutation in the protease (PR) gene involves a GAT to AAT mutation that is well-characterized and specific to the protease inhibitor nelfinavir (NFV) [22, 23]. The D30N has been identified to biochemically alter viral protease activity in heterologous cleavage studies [7, 13, 18, 23-25]. The $\mathrm{M} 184 \mathrm{~V}$ mutation in the reverse transcriptase (RT) gene, which causes primary resistance to lamuvidine (3TC), has been extensively studied and is common in treated patients $[6,26-28]$. Viral isolates with the M184V mutation in RT 
have lower RC than viral isolates without the mutation [17]. In one study, HIV infected individuals with significant ART drug resistance were randomized to receive $3 \mathrm{TC}$ or to stop ART completely; those receiving 3TC alone experienced a lower increase in viral load, a slower rate of decline in CD4 $\mathrm{T}$ cell percentage, and fewer adverse clinical events related to HIV infection [26].

Assessing the relative impact of mutations on HIV fitness is difficult because there are often many more parameters than there are data. Previously we described an informaticsbased method to identify the relative fitness cost of mutations in protease using a Bayesian hierarchical model [29]. Using this model we found mutations in protease that had a relatively large decrease in viral RC. However, it is unknown if the in silico results correspond to an actual in vivo fitness decrease.

The effect of mutations on viral fitness and pathogenicity often cannot be determined in vitro; however, the SCID-hu mouse model allows direct assessment in human tissue in an in vivo setting. The SCID-hu mouse model of HIV infection is well described and has been used to examine the mechanisms of viral pathogenesis in primary human lymphoid tissue $[8,30]$. We and others have found that HIV-1 directly injected into human thy/liv implants in SCID-hu mice results in reproducible infection and severe depletion of human cells bearing the CD4 molecule [30-32]. Stoddart et al. [8] examined viral fitness in 8 clinical isolates compared with $\mathrm{HIV}_{\mathrm{NL4}-3}$, however none of these isolates contained the D30N mutation. They determined that while the RC of PI-resistant strains of HIV-1 in peripheral blood mononuclear cells (PBMCs) was moderately impaired compared to wild-type (WT) virus, the RC of PI-resistant strains in vivo was highly impaired. The observation that differences in replication between PI-resistant strains and WT stains were seen in vivo in the SCID-hu mouse and not in vitro in PBMCs indicates that the SCID-hu mouse is an excellent model system to assess the fitness of viruses with different antiretroviral drug resistance mutations in primary human tissue. In contrast to other studies with patient isolates $[8,33]$, we explicitly tested specific mutations found a priori to have a fitness effect. In the current study, we utilized results from a bio-informatics model and then utilized in vitro tissue culture and the in vivo SCID-hu mouse model to determine if this mutation confers a biological fitness defect. This study demonstrates the synergistic possibilities in translational research and validates a unique drug resistance mutation in HIV-1 that confers an in vivo fitness defect in the virus.

\section{METHODS}

\section{Bioinformatics Analysis}

We used a Bayesian hierarchical model to determine the relative replication capacity effect of mutations in 161 genotype-phenotype pairs of HIV-1 protease (described in [29]). Because we have a much greater number of parameters than data points (large $\mathrm{p}$, small $\mathrm{n}$ ), prior specification is critically important. Fortunately, there exists a wealth of information elucidating links between HIV replication and specific mutations based on site-directed mutagenesis and drug-resistance studies. Bayesian methods allow us to explicitly take into account what is known a priori about mutational patterns in HIV-1. The idea is to provide priors with both shrinkage and variable selection components. Let $y_{i}$ be the continuous fitness phenotype for the $i^{\text {th }}$ HIV-1 sequence $\left(Y=\left(y_{1}, \ldots, y_{n}\right)^{\prime}\right)$. Let $x_{i j}$ represent the $j^{\text {th }}$ codon position for the $i^{\text {th }}$ sequence $\left(X_{j}=\left(x_{1 j}, \ldots, x_{n j}\right)^{\prime}\right)$. In this case the $x_{j}$ 's are $0 / 1$ indicators of a mutation away from wild-type at each codon position along the protease genome. The informatics problem is to find the set of $X_{j}$ 's that are contributing to the fitness phenotype. Because the parameter space is much larger than the number of sequences, backwards and forward selection using conventional regression methods are unlikely to yield useful results. Kuo and Mallik [34] created a class of prior that has both a shrinkage and a selection component where all coefficients are exchangeable. Following Kuo and Mallik the prior parameters are specified by assuming all regression coefficients have the same prior. There are two parts to this prior, the $\delta_{j}$ is the model selection part and the prior on the regression coefficient $\beta_{j}$ is the shrinkage component. $\beta_{j}$, the prior mean and variance of the regression coefficient, is modeled as a Normal with hyperpriors on the mean and variance which are fixed. The $\delta_{j},\left(\delta_{j} \sim \operatorname{Bernoulli}(\rho)\right)$, is a binary indicator function for the presence of a fitness effect on the regression coefficient, (which then becomes $\beta_{j} \delta_{j}$ ) in the model. This prior is the same for all regression coefficients and thus all of the $x_{i j}$ 's are exchangeable. Fig. (1A) illustrates this prior. This model is scientifically uninformative in that each codon position has the same prior effect on fitness. We call this Model 1, the uninformative prior.

Site-directed mutagenesis and HIV-1 drug-resistance studies have gathered a wealth of information on identifying codon positions that appear to have an in vitro effect on fitness. To incorporate this information into our model we generalize our KM priors to allow for subsets of codon positions whose effects are exchangeable within the subset (the Exchangeable on Subsets Prior (ESP)). That is codons within the same set have the same prior while codons in different subsets have different priors. To see how this might work, we could allow a set of codons that site-directed mutagenesis has implicated as having a fitness effect to have a higher probability of inclusion (higher $\rho$ ) and a higher prior mean fitness effect (through the mean and variance hyperparameters of $\beta_{j}$ ) that codons not in this set. All of the codons in this selected subset are exchangeable, i.e. have the same prior. However, codons that are not in this subset could have a prior $\delta_{j}$ that has a lower probability of inclusion, $\rho$, and a smaller fitness effect, $\beta_{j}$. Fig. (1B) illustrates this ESP prior for when there are 4 different subsets. However the scientific literature uses different methods to determine importance and although there is general agreement for certain codon positions, there is not a consensus for all 
positions. Because of this, we create 3 ESP priors based on 3 relevant papers in the HIV-1 literature.

(A)

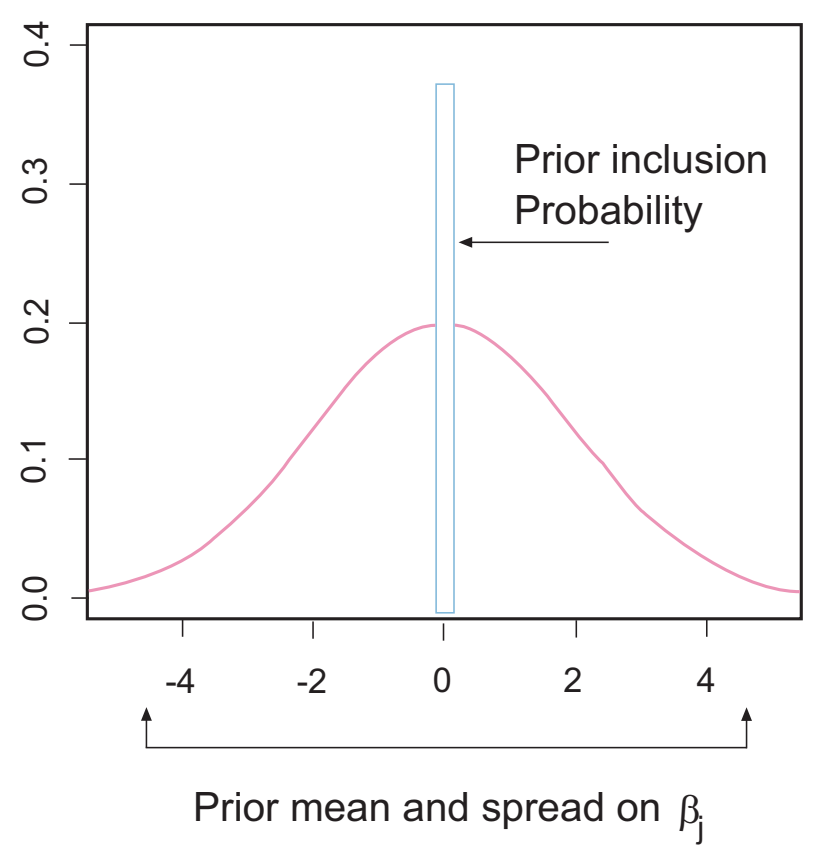

(B)

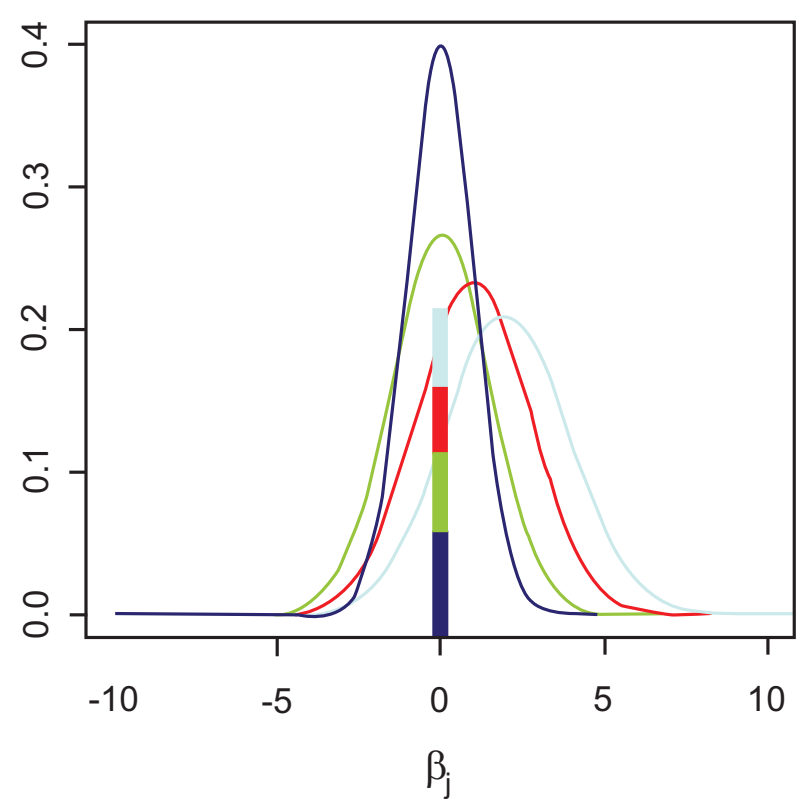

Fig. (1). Prior illustration for the models. For each codon position $\mathrm{j}$, the column shows $\delta_{j}$, the prior $0 / 1$ indicator if codon $j$ is important to the fitness phenotype. The curve shows the prior distribution on the regression coefficient $\beta_{j}$. (A) Model 1, the uninformative prior. All codon positions have the same prior. (B) The Exchangeable on Subsets Prior (ESP). In the figure, 4 subsets are shown. Each codon within a subset has the same prior $\delta_{j}$ and prior distribution on $\beta_{j}$. Different subsets have different values for the priors on the $\delta_{j}$ 's and $\beta_{j}$ 's. The four subsets are shown in different colors.
Specifically, we created a prior based on the paper by Swanstrom and Erona [35], a prior based on the paper by Foulkes and DeGruttola [36], and one based on Loeb et al. [18]. For each paper a model is constructed in which all codon positions identified as having a fitness effect are deemed important. These codon positions are all given the same prior with high inclusion probability and higher prior fitness effects. Codons that are not identified in the paper are deemed unimportant and are all given priors with low values of rho and diffuse $\beta_{j}$ 's centered around zero. To assess the consensus of positions we created another ESP prior where each model (the 3 literature based models and the uninformative Model 1) "voted" for a specific codon to be included in the "important" set and that codon was weighted according to the number of votes. In the voting model there were 4 classes of priors according to the number of votes a codon position received: $0,1,2,3$ or 4 . Codons with more votes had a higher probability of inclusion, and beta coefficients with larger fitness effects.

To choose which prior fit the data best we used Prior Model Selection (PMS). In brief, we used Gibbs sampling to generate draws from the posterior distribution $f(\beta, \delta \mid y)$ of each of the 5 models. The marginal likelihood of $\mathrm{Y}$, $m(y)=f(y \mid \theta) \pi(\theta) / \pi(\theta \mid y)$, was calculated using Chib's [37] method; where $\theta$ represents the parameters and $\pi$ represents the prior. Each model was run 10 times to obtain an estimate of the standard deviation of the log marginal likelihood for each model. Bayes Factors were calculated from the difference of the average log marginal likelihood values and were used to compare the models. To determine the in vivo effect in the SCID-hu mouse model, we conducted a power analysis to determine the number of mice that would be required in each group. The study was powered to detect a difference in the D30N groups (D30N and $\mathrm{D} 30 \mathrm{~N}+\mathrm{M} 184 \mathrm{~V}$ ) versus control (wild-type). The effect size of the power calculations were based on the results of the statistical model presented in Kitchen et al. [29]. For each mouse, the cumulative area under the curve for log HIV-1 RNA viral burden and total CD4+ thymocyte count were calculated. Groups were compared using the two-sided Wilcoxon Rank Sum Test. Groups were also compared over time using a nonlinear mixed effects model. Group by time comparisons were made using the Wilcoxon Rank Sum test with p-values adjusted for the overall type 1 error rate using a Bonferroni correction.

\section{Generation of Drug-Resistant Viral Stock}

Viral mutants were generated by the introduction of the $\mathrm{D} 30 \mathrm{~N}$ and $\mathrm{M} 184 \mathrm{~V}$ mutations into a molecularly cloned strain of HIV $\left(\mathrm{HIV}-1_{\mathrm{NL} 4-3}\right)$ by site-directed mutagenesis. Viral stocks were prepared by electroporation of CEMx 174 cells with plasmid DNA encoding the genome of wild-type and mutant viruses. Virus was harvested in culture supernatant 2 and 3 days following electroporation and quantitation of p24 gag was performed by enzyme-linked immunosorbent assay (ELISA, Coulter, Hialeah, Florida). Viral titers were determined through a standard limiting dilution assay on CEMx174 cells. 


\section{In Vitro Viral Growth}

Freshly isolated peripheral blood mononuclear cells (PBMC) were obtained in the form of leukopacks from anonymous donors by the UCLA AIDS Institute Virology Core in accordance with IRB protocols. PBMCs were purified by Ficol purification and cells were then stimulated for three days with phytohemagglutin (PHA)(1 microgram $/ \mathrm{ml}$ (Sigma) and IL-2 (100 units $/ \mathrm{ml})(\mathrm{R} \& D$ Systems, Minneapolis, MN). Cells were then infected, separately, with each indicated virus at a multiplicity of infection (MOI) of 0.003 in a volume of $1 \mathrm{ml}$ for two hours at 37 degrees. Cells were then cultured at a concentration of $1 \mathrm{x} 10^{6} / \mathrm{ml}$ and at the indicated times 100 microliters of supernatant was removed and replaced with fresh medium. Viral supernatant was then placed in PBS containing 1\% Triton X-100 and p24 levels were quantitated by ELISA, as described above.

\section{SCID-hu Mice}

SCID-hu thy/liv mice were constructed by implanting human fetal thymus and liver under the kidney capsule of C.B.17 SCID mice as described [28, 34]. Thy/liv implants $(\mathrm{n}=5$ mice per group) were infected by direct injection of 100 infectious units of either wild type HIV-1 $1_{\text {NL4-3 }}$ or HIV-1 ${ }_{\text {NL4-3 }}$ containing the $\mathrm{D} 30 \mathrm{~N}$, the $\mathrm{M} 184 \mathrm{~V}$, or both the $\mathrm{D} 30 \mathrm{~N}$ and M184V mutations or were mock infected with medium alone. At the specified time points, thy/live implants were biopsied utilizing survival surgery procedures as described $[28,34]$.

\section{Flow Cytometry}

Single cell suspensions were made from the biopsied tissue and analyzed by flow cytometry for the expression of CD45, CD3, CD4, and CD8 similar to that described [35, 36]. Cells were run on a Coulter FC500 (Coulter, Hialeah, FL) flow cytometry and data was analyzed by FlowJo software (Treestar, Ashland, OR). Depletion of CD4+ cells (CD4+CD8+ and CD4+CD8- thymocytes) was determined by comparison of cells from HIV infected implants to mockinfected controls.

\section{Quantitative DNA PCR}

A fraction of cells was removed and DNA was purified as previously described $[38,39]$. Quantitation of proviral and cellular DNA was performed with real time quantitative PCR using primers specific for human beta globin sequences and full-length HIV reverse transcripts (the long terminal repeatgag junction) as described by [40].

\section{RESULTS}

Utilizing a data set of 161 of genotype/phenotype pairs (described in [29]), we used a Bayesian hierarchical model to determine the relative effects of mutation in silico. The priors that were used included an uninformative prior across the whole protease genome, three literature based ESP priors and a voting ESP prior whereupon each model "voted" for a codon position. We then incorporated prior model selection (PMS) to choose among our priors. The voting prior had the smallest log marginal likelihood of all the models and testing the uninformative model versus the voting model decisively rejected the uninformative model (log Bayes Factor=91.32). Using this model as our final model, we were able to assess the relative cost of each mutation in protease, in terms of $\mathrm{RC}$. The model found that the D30N mutation had the largest decrease in RC relative to other resistance-associated mutations suggesting that this mutation may have clinical benefit in prolonging disease progression by conferring a RC defect. Table 1 lists the regression coefficient estimates and the $95 \%$ credible interval of the top 5 drug-resistance associated mutations in protease.

Table 1. The Estimated Fitness Effect, $E[\beta \delta \mid y]$ and the Corresponding 95 Percent Credible Interval for the Top 5 Drug-Resistance Associated Mutations from the Final Best Fitting Model

\begin{tabular}{|c|c|c|}
\hline $\begin{array}{c}\text { Codon } \\
\text { Position }\end{array}$ & $\begin{array}{c}\text { Estimated Fitness } \\
\text { Effect }\end{array}$ & $\begin{array}{c}\text { 95\% Credible } \\
\text { Interval }\end{array}$ \\
\hline \hline $\mathrm{D} 30 \mathrm{~N}$ & -16.72 & $(-30.32,-2.92)$ \\
\hline $\mathrm{V} 77 \mathrm{I}$ & -13.49 & $(-22.34,-4.62)$ \\
\hline $\mathrm{V} 82 \mathrm{~A} / \mathrm{F} / \mathrm{T} / \mathrm{S}$ & -16.61 & $(-25.40,-5.35)$ \\
\hline $\mathrm{I} 84 \mathrm{~V} / \mathrm{A} / \mathrm{C}$ & -13.11 & $(-23.28,-0.81)$ \\
\hline $\mathrm{L} 90 \mathrm{M}$ & -12.60 & $(-23.25,-1.91)$ \\
\hline
\end{tabular}

To assess the effects the in silico identified D30N PR and the previously implicated M184V RT mutation in infectivity of HIV-1 we assessed viral titers of molecularly clones variants of $\mathrm{HIV}-1_{\mathrm{NL4}-3}$ containing these mutations in a standard limiting dilution assay. HIV- $1_{\mathrm{NL} 4-3}$ wild type, HIV$1_{\mathrm{NL} 4-3}$ with the D30N mutation, HIV- $1_{\mathrm{NL} 4-3}$ with the M184V mutation, and HIV-1 $1_{\mathrm{NL} 4-3}$ containing both the D30N and M184V mutations all had a titer of 300 picograms per infectious unit, indicating that the presence of the mutation in the drug resistant viruses did not affect initial viral infectivity. We then compared the ability of theses viruses to replicate in PHA-activated PBMCs. Cells were infected at a relatively low multiplicity of infection (MOI) to detect and magnify differences in the ability of the virus to replicate over multiple rounds of infection. We found that the virus containing the M184V mutation displayed slightly delayed replication kinetics while the viruses containing the D30N alone and in combination with $\mathrm{M} 184 \mathrm{~V}$ and the double mutant had a more dramatic decrease in viral replication as compared to the wild-type virus (Fig. 2). In all, these data indicate that mutations do not alter the infectivity of the virus, but reduce viral RC.

To examine the effects of these mutations on viral fitness and pathogenesis in vivo, we examined the accumulation of HIV DNA and the level of virus-induced CD4+ thymocyte depletion over time using the SCID-hu mouse model. Thy/liv implants were initially infected with equivalent amounts of infectious units of wild type, D30N-containing, M184V-containing, and both $\mathrm{D} 30 \mathrm{~N}$ and M184V containing HIV-1 $1_{\text {NLA-3 }}$ in parallel and the effects of the virus were examined at 3,5, and 7 weeks following infection. Proviral DNA was detected in mice infected with each different virus within three weeks post infection (Fig. 3). Infection of thy/liv implants with wild type (NL4-3) produced time dependent increases in log HIV-1 DNA levels. Infection of mice with $\mathrm{HIV}-1_{\mathrm{NL} 4-3}$ containing the $\mathrm{M} 184 \mathrm{~V}$ mutation produced similar viral kinetics as the wild type virus. The 
average cumulative area under the curve was not significantly different for $\mathrm{M} 184 \mathrm{~V}$ versus wildtype. Mice infected with HIV-1 $1_{\mathrm{NL} 4-3}$ containing the D30N or $\mathrm{D} 30 \mathrm{~N}+\mathrm{M} 184 \mathrm{~V}$ mutation, however, had barely detectable viral DNA at all three time points. Mice harboring HIV-1 $1_{\text {NL4- }}$ ${ }_{3}$ containing the D30N mutant strains had significantly lower levels of HIV RNA than the wildtype or M184V alone groups $(\mathrm{P}=0.001)$. There was no statistically significant difference in HIV DNA levels between the D30N and the $\mathrm{D} 30 \mathrm{~N}+\mathrm{M} 184 \mathrm{~V}$ infected group at any time point.

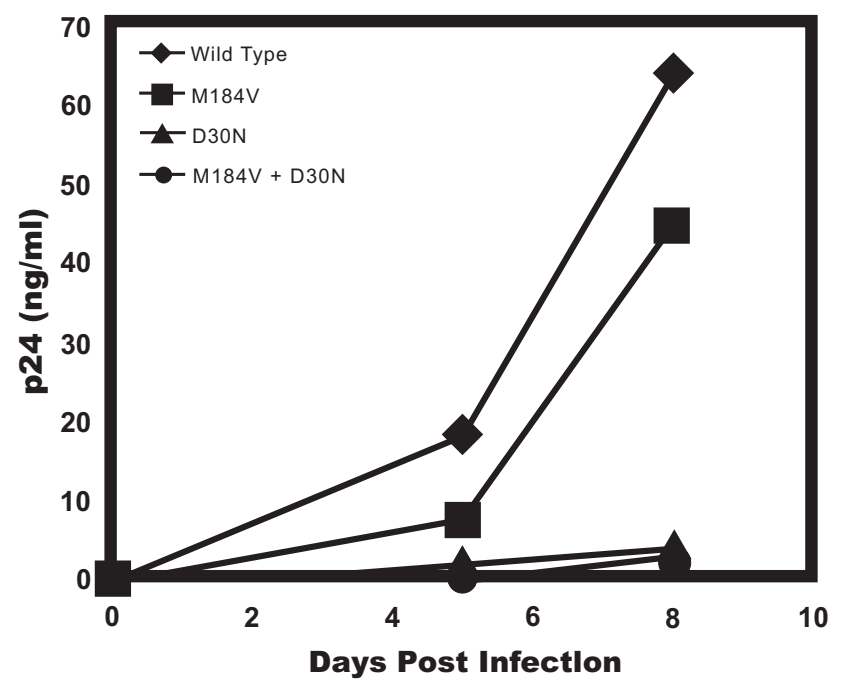

Fig. (2). Replication kinetics of wild-type, M184V-containing, D30N-containing, and M184V and D30N-containing viruses in PHA-stimulated PBMC. Viral p24 antigen production was assessed at days 5 and 8 post infection. The results shown are representative of 3 independent experiments with PBMC derived from different donors.

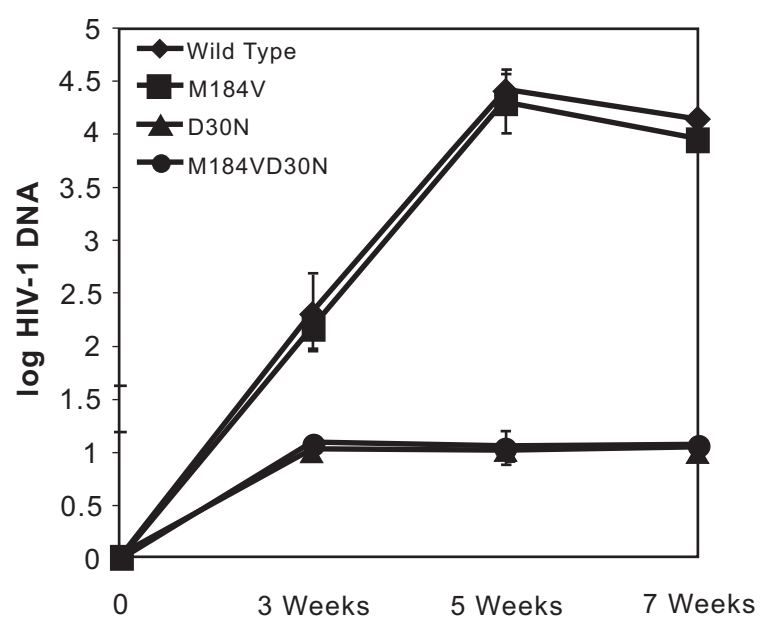

Fig. (3). Viral replication of wild-type, M184V-containing, D30Ncontaining, and $\mathrm{M} 184 \mathrm{~V}$ and $\mathrm{D} 30 \mathrm{~N}$-containing viruses in vivo. Thy/liv implants were infected, separately ( $\mathrm{n}=5$ mice per group), with identical amounts of infectious units of each virus. Implants were analyzed following biopsy of infected tissue by quantitative PCR for HIV proviral DNA at 3, 5, and 7 weeks following infection. The amounts of proviral DNA are provided as copies of full length proviral DNA per 100,000 cells, as determined by the use of HIV and human $\beta$-globin specific primers and quantitative comparison to known controls. Mock infected controls were negative for HIV proviral DNA (not shown).

Cellular depletion of CD4 bearing thymocytes which is indicative of viral pathogenesis, primarily in the CD4+CD8+ population, was observed in mice infected with wild type HIV-1 NL4-3 and the M184V containing strains within 5 weeks following infection (Fig. 4). Seven weeks post inoculation, we found profound CD4+ thymocyte depletion in the mice infected with wild-type virus or the virus containing the M184V mutation alone. The level of depletion in these two groups was significantly greater than the level of depletion found in mice infected with strains that included the $\mathrm{D} 30 \mathrm{~N}$ mutation $(\mathrm{p}=0.001)$. In fact, mice infected with strains containing the D30N mutation (including the double mutant) were not statistically different from the mock infected mice in terms of percent total CD4+ thymocytes. The differences in percent total thymocytes between mock and the two D30N groups were not significant at any time point (even without adjusting for multiple comparisons). Mice infected with the M184V mutation alone had a profound depletion in thymocytes compared to mock infected mice $(p=0.027)$ and was not significantly different from the depletion found in mice infected with wildtype virus, indicating that the $\mathrm{D} 30 \mathrm{~N}$ is primarily responsible for the attenuated virulence.

\section{DISCUSSION}

These results illustrate the utility of biostatisticians and biologists working together and demonstrate the synergy possible with translational studies. This work was prefaced by a thorough examination of protease mutations in a bioinformatics system whereby the D30N mutation was found to have a profound effect on in vitro replication. To be able to specifically attribute viral attenuation with the mutation, we constructed point mutants instead of using patient isolates that may have accrued mutations at other loci in the viral genome. Our results indicate that the D30N mutation has a substantial effect on the ability of HIV-1 to deplete thymocytes and to replicate in an in vivo system.

Our data clearly demonstrate that mutations in the HIV-1 genome may differ greatly in their impact on HIV replication capacity and pathogenicity. Whereas the virus containing the M184V mutation alone conferred a RC defect in vitro, we did not find evidence for decreased pathogenicity of this virus in vivo. In our experiments, mice infected with this mutant did not have statistically different viral loads or CD4+ thymocyte counts than mice infected with wild-type. In addition, the M184V did not enhance or inhibit viral replication or the ability of the virus to deplete thymocytes when coupled with the D30N mutation. There was no statistically significant difference between mice infected with the D30N mutant and those infected with the $\mathrm{D} 30 \mathrm{~N}+\mathrm{M} 184 \mathrm{~V}$ double mutant at any time point. These studies demonstrate that $\mathrm{RC}$ results observed in vitro do not necessarily correlate with a defect in viral fitness in vivo.

Our results contrast from those described by Stoddard et al. who found that mice infected with a virus (210P) containing protease mutation at I54V and V82A had a higher level of viremia than mice infected with wild-type virus. However, comparison is difficult due to the presence of different mutations in the HIV protease (D30N versus 
A.

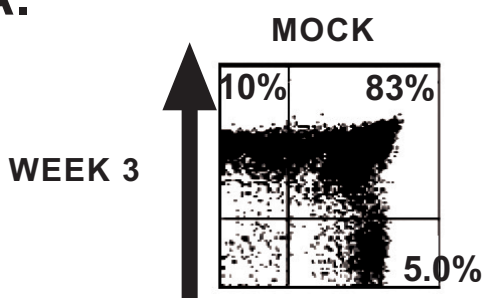

WEEK 5

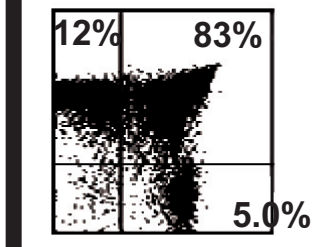

M184V
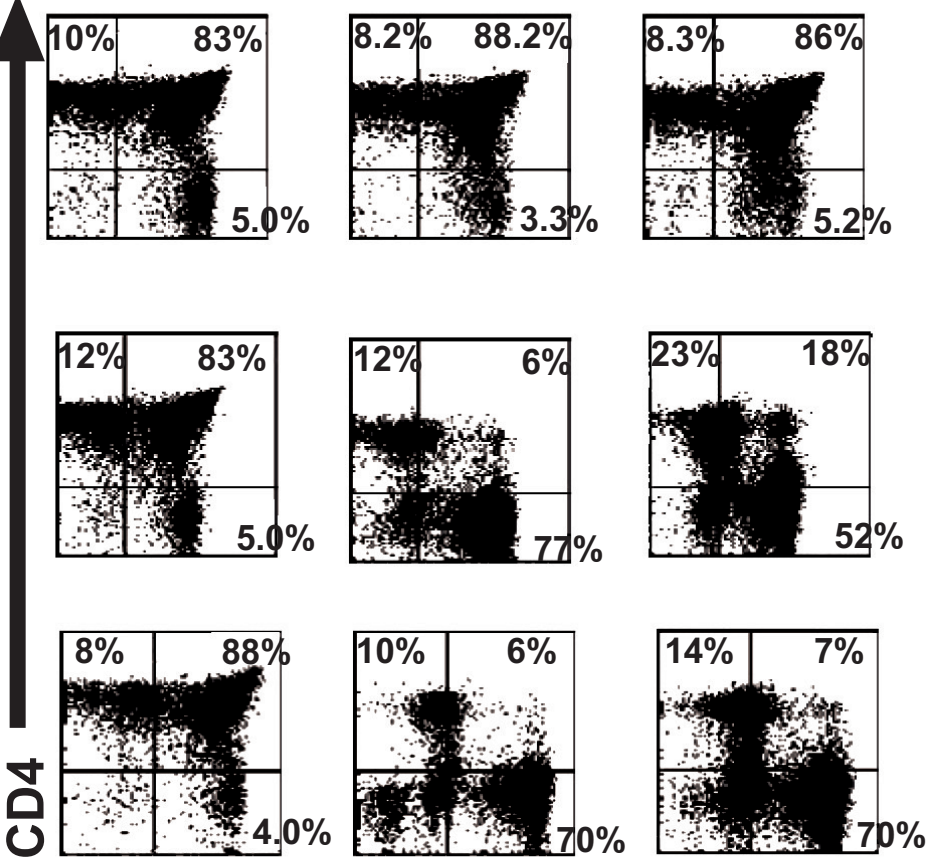

D30N

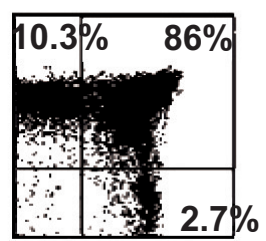

M184V+

D30N
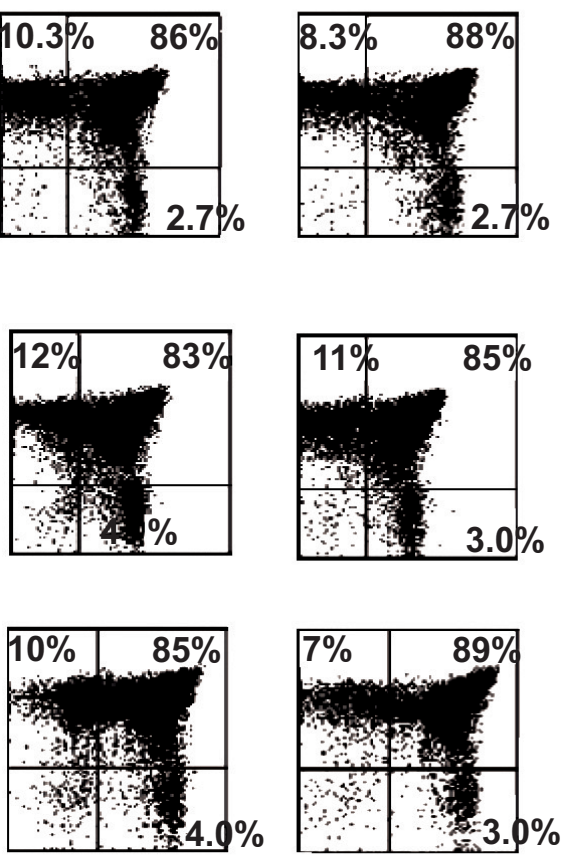

\section{CD8}

B.

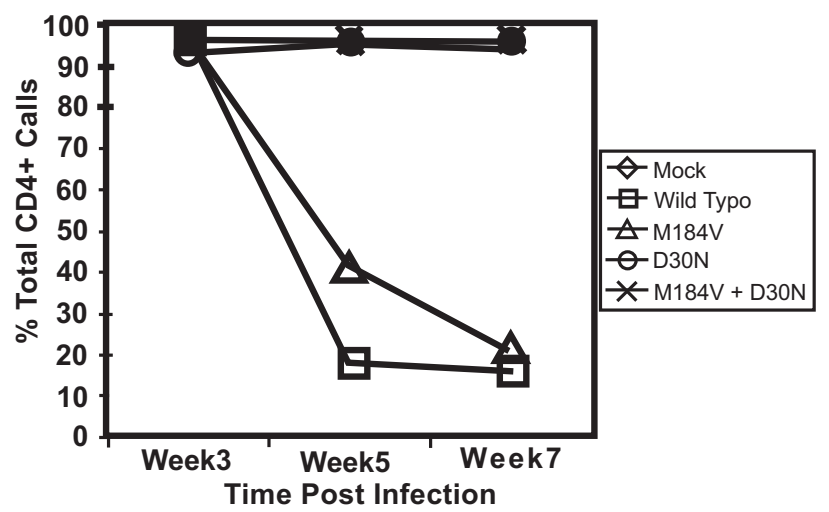

Fig. (4). Depletion of CD4-bearing cells following infection with wild-type, M184V-containing, D30N-containing, and M184V and D30Ncontaining viruses in vivo. (A) CD4 versus CD8 flow cytometry staining profiles were determined on biopsied thy/liv tissue at the indicated times following infection with one of the four indicated viruses. Cells were stained with monoclonal antibodies (and their corresponding fluorochromes) to CD45 (FITC), CD8 (ECD), and CD4 (APC). CD4 and CD8 profiles of this population are given by gating on the human CD45+ population of cells. The percentages of cells in each quadrant are indicated. The data is representative of one mouse per group (total of 5 mice per group) of a total of three experiments. (B) Graphic representation of the percentage of total CD4+ cells in the respective thy/liv implants over time.

$\mathrm{I} 54 \mathrm{~V}+\mathrm{V} 82 \mathrm{~A}$ ), as well as the variability inherent in experiments employing SCID-hu mice in which a chimeric organ is created by engraftment of primary human tissues. Nonetheless, the differences in these results may also suggest that virus containing the $\mathrm{D} 30 \mathrm{~N}$ mutation is less able to replicate in the thymus than virus containing the V82A mutation (as is suggested by our bioinformatics model). The D30N mutation was chosen as it had the largest decrease in relative replication capacity. It is noteworthy that previous studies have shown that the V28A and the D30N mutations do no co-occur in the same genome [41-43].

Although some patients experience the so called "discordant state" characterized by having both high viral loads and stable CD4+ T-cell counts, but there are many others who are concordant and have high viral loads and 
decreasing CD4 counts despite the presence of PI resistance mutations [5, 16, 44-46]. The latter state likely arises when HIV acquires compensatory mutations that increase its level of fitness and restore its ability to deplete CD4+ T-cells. The apparent lack of pathogenicity of the HIV-1 D30N mutant suggests that this mutation may represent a genetic "deadend" for the virus. Once the D30N mutation has been acquired, the virus may not be able to replicate well enough to return to wild-type fitness. There may be other genetic bottlenecks in the virus that can be capitalized upon to drive the virus into an unfit-state and preserve CD4+ T-cells, however further research is needed.

In people infected with clade B strains of HIV, those that harbor virus containing the $\mathrm{D} 30 \mathrm{~N}$ mutation often also have the L63P mutation. The L63P mutation was not identified as having a large fitness defect by our model, likely because the L63P mutation is prevalent in patients who are treatment naïve and is therefore not a drug-resistance mutation. Moreover, others have reported that the replication of a D30N containing variant was not significantly different from a L63P+D30N dual mutant [7]. Similarly, patients with the D30N mutation often develop the N88D/S mutation. N88D/S did not have a significant effect on fitness in the statistical model and was not tested. HIV variants with both the D30N and N88D mutation was also found to have decreased replication capacity in vitro, including subtype C strains [24, $47,48]$.

There are several limitations to this study. Our analysis was based on examinations of mutations found in protease in HIV-1 clade B strains and our biologic experiments used viruses based on an HIV-1 clade B strain. Although the $\mathrm{D} 30 \mathrm{~N}$ has been identified as having an effect in vitro in HIV-1 clade C strains, it not known if the in vivo result is generalizable to other non-B clades and HIV-2. It is also possible that the $\mathrm{D} 30 \mathrm{~N}$ mutation might not be preferentially selected through nelfinavir treatment in non clade-B strains. Further there could be unaccounted variation in the human tissues used in the SCID-hu model that limits the generalizability of this finding. Further research needs to be done.

In conclusion, our results suggest the possibility of the existence of genetic bottlenecks in HIV-1 that select for mutations that diminish replication capacity and in vivo fitness to such an extent that the virus is unable to acquire compensatory mutations as well as deplete target cells. Further work is needed to find other possible dead-end mutations in regions of the genome that are targeted by other antiretroviral agents now under development or in initial use, such as inhibitors of the strand transfer activity of the HIV integrase protein. In all, this study validates a bioinformatics model tested by an in vivo system.

\section{ACKNOWLEDGEMENTS}

This work was supported by the Center for AIDS Research UCLA AI07 (CMRK and SK) and the NIAID to PK (AI01996). Effort by PK was also supported by an Elizabeth Glaser Pediatric AIDS Foundation Scientist Award.

\section{REFERENCES}

[1] Ho DD, Neumann AU, Perelson AS, Chen W, Leonard JM, Markowitz M. Rapid turnover of plasma virions CD4+ lymphocytes in HIV-1 cells. Nature 1995; 373: 123-6.

[2] Wei X, Ghosh SK, Taylor ME, et al. Viral dynamics in HIV-1 infection. Nature 1995; 373: 117-22.

[3] Perelson AS, Essunger P, Ho DD. Dynamics of HIV-1 and CD4+ lymphocytes in vivo. AIDS 1997; 11(Suppl A): S17-S24.

[4] Mammano F, Petit C, Clavel F. Resistance-associated loss of viral fitness in HIV-1: phenotypic analysis of protease gag coevolution in protease-inhibitor treated patients. J Virol 1998; 72: 7632-7.

[5] Deeks SG, Barbour JD, Martin JN, Swanson MS, Grant R. Sustained CD4+ T-cell response after virologic failure of proteasebased regimens in patients with HIV infection. J Infect Dis 2000; 181: $946-53$.

[6] Dykes C, Demeter L. Clinical significance of human immunodeficiency virus type 1 replication fitness. Clin Microbiol Rev 2007; 20(4): 550-78.

[7] Martinez-Picado J, Savara AV, Sutton L, D'Aquila RT. Replicative fitness of protease inhibitor-resistant mutants of HIV-1. J Virol 1999; 73: 3744-52.

[8] Stoddart CA, Leigler TJ, Mammano F, et al. Impaired replication of protease resistant HIV-1 in human thymus. Nat Med 2001; 7: 712-8.

[9] Deeks SG. Virologic outcomes with protease inhibitor therapy in an urban AIDS clinic: relationship between baseline characteristics and response to both initial and salvage therapy. AIDS 1999; 13: F34-F44.

[10] Fatkenheuer G, Theisen A, Rockstroh J, et al. Virologic treatment failure of protease inhibitor therapy in an unselected cohort of HIVinfected patients. AIDS 1997; 11: F113-6.

[11] Ledergerber B, Egger M, Opravil M, et al. Clinical progression and virologic failure on highly active antiretroviral therapy in HIV-1 patients: a prospective cohort study. Lancet 1999; 353: 863-8.

[12] Lucas GM, Chaisson RE, Moore RD. Highly active antiretroviral therapy in a large urban clinic: risk factors for virologic failure and adverse drug reactions. Ann Intern Med 1999; 131: 81-7.

[13] Nijhuis $M$, Deeks S, Boucher C. Implications of antiretroviral resistance on fitness. Curr Opin Infect Dis 2001; 14: 23-8.

[14] Deeks SG, Wrin T, Leigler TJ, et al. Virologic and immunologic consequences of discontinuing combination antiretroviral-drug therapy in HIV-infected patients with detectable viremia. N Eng J Med 2001; 344(7): 472-80.

[15] Deeks SG, Grant RM, Wrin T, et al. Persistence of drug-resistant HIV-1 after structured treatment interruption and its impact on treatment response. AIDS 2003; 17: 361-70.

[16] Deeks SG, Barbour JD, Grant RM, Martin JN. Duration and predictors of CD4 T-cell gains in patients who continue combination therapy despite detectable plasma viremia. AIDS 2002; 16: 201-7.

[17] Barbour JD, Wrin T, Grant RM, et al. Evolution of phenotypic drug susceptibility and viral replication capacity during long-term virologic failure of protease inhibitor therapy in human immunodeficiency virus-infected adults. J Virol 2002; 76(21): 11104-12.

[18] Loeb DD, Swanstrom R, Everitt L, Manchester M, Stamper SE, Hutchison CA. Complete mutagenesis of the HIV-1 protease. Nature 1989; 340(6232): 397-400.

[19] Mammano F, Petit C, Clavel F. Retracing the evolutionary pathways of HIV-1 resistance to protease inhibitors: viral fitness in the absence in the presence of drug. J Virol 2000; 74: 8524-31.

[20] Nijhuis M, Schuurman R, DeJong D, et al. Increased fitness of drug resistant HIV-1 protease as a result of acquisition of compensatory mutations during suboptimal therapy. AIDS 1999; 13: 2349-59.

[21] Penn ML, Myers M, Eckstein DA, et al. Primary recombinant HIV1 strains resistance to protease inhibitors are pathogenic in mature human lymphoid tissues. AIDS Res Hum Retroviruses 2001; 17: 517-23.

[22] Patick AK, Duran M, Cao Y, et al. Genotypic and phenotypic characterization of human immunodeficiency virus type 1 variants isolated from patients treated with the protease inhibitor nelfinavir. Antimicrob Agents Chemother 1998; 42(19): 2637-44. 
[23] Perrin V, Mammano F. Parameters driving the selection of nelfinavir-resistant human immunodeficiency virus type 1 variants. J Virol 2003; 77(18): 10172-5.

[24] Sugiura W, Matsuda Z, Yokomaku Y, et al. Interference between D30N and L90M in selection and development of protease inhibitor-resistant human immunodeficiency virus type 1 . Antimicrob Agents Chemother 2002; 46(3): 708-15.

[25] Devereux HL, Emery VC, Johnson MA, Loveday C. Replicative fitness in vivo of HIV-1 variants with multiple drug-resistance associated mutations. J Med Virol 2001; 65: 218-24.

[26] Castagna A, Danise A, Menzo S, et al. Lamivudine monotherapy in HIV-1 infected patients harboring a lamivudine-resistance virus: a randomized pilot study (E-184V study). AIDS 2006; 20(6): 795803.

[27] Gallant JE. The M184V mutation: what it does, how to prevent it, and what to do with it when it's there. AIDS Read 2006; 16(10): 556-9.

[28] Paredes R, Cheng I, Kuritzkes D, Tuomala R, Group WaITSW. Postpartum antiretroviral drug resistance in HIV-1 infected women receiving pregnancy-limited antiretroviral therapy. AIDS 2010; 24(1): 45-53.

[29] Kitchen C, Weiss R, Liu G, Wrin T. HIV-1 viral fitness estimation using exchangeable on subset priors and prior model selection. Stat Med 2007; 26(5): 975-90.

[30] Stoddart CA, Bales C, Bare J, et al. Validation of the SCID-hu Thy/Liv mouse model with four classes of licensed antiretrovirals. PLoS One 2007; 2(7): e655.

[31] Aldrovandi GM, Feuer G, Gao L, et al. The SCID-hu mouse as a model for HIV-1 infection. Nature 1993; 363(6431): 732-6.

[32] Kitchen S, Zack J. HIV type 1 infection in lymphoid tissue: natural history and model systems. AIDS Res Hum Retroviruses 1998; 14(Suppl 3): S235-9.

[33] Picchio G, Valdez H, Sabbe R, et al. Altered viral fitness of HIV-1 following failure of protease inhibitor-based therapy. J Acquir Immune Defic Syndr 2000; 25: 289-95.

[34] Kuo L, Mallick B. Variable selection for regression models. Sankya B 1999; 60: 65-81.

[35] Swanstrom R, Erona J. HIV-1 protease inhibitors: therapeutic successes failures, suppression, resistance. Pharmacol Ther 2000; 86: $145-70$.

[36] Foulkes AS, DeGruttola V. Characterizing the relationship between HIV-1 genotype and phenotype: prediction based classification. Biometrics 2002; 58: 145--56.
[37] Chib S. Marginal likelihood from the Gibbs output. J Am Stat Assoc 1995; 90: 1313-21.

[38] Kitchen SG, Uittenbogaart CH, Zack JA. Mechanism of HIV-1 localization in CD4-negative thymocytes: differentiation from a CD4-positive precursor allows productive infection. J Virol 1997; 71(8): 5713-22.

[39] Zack JA, Arrigo SJ, Weitsman SR, Go AS, Haislip A, Chen IS. HIV-1 entry into quiescent primary lymphocytes: molecular analysis reveals a labile, latent viral structure. Cell 1990; 61(2): 213-22.

[40] Gorry PR, Bristol G, Zack JA, et al. Macrophage tropism of human immunodeficiency virus type 1 isolates from brain and lymphoid tissues predicts neurotropism independent of coreceptor specificity. J Virol 2001; 75(21): 10073-89.

[41] Hoffman N, Schiffer C, Swanstrom R. Covariation of amino acid positions in HIV-1 protease. Virology 2003; 314: 536-48.

[42] Wu TD, Schiffer C, Gonzales MJ, et al. Mutation patterns and structural correlates in HIV-1 protease following varying degrees of protease inhibitor treatment. J Virol 2003; 77: 4836-47.

[43] Sugiura W, Matsuda Z, Yokomaku Y, et al. Interference between $\mathrm{D} 30 \mathrm{~N}$ and $\mathrm{L} 90 \mathrm{M}$ in selection and development of protease inhibitor-resistant human immunodeficiency virus type 1 . Antimicrob Agents Chemother. 2002; 43(3): 708-15.

[44] Deeks S, Hoh R, Neilands TB, et al. Interruption of treatment with individual therapeutic drug classes in adults with multidrug resistant HIV-1 infection. J Infect Dis 2005; 192: 1537-44.

[45] Mezzaroma I, Carlesimo M, Pinter E, et al. Clinical and immunologic response without decrease in virus load in patients with AIDS after 24 months of highly active antiretroviral therapy. Clin Infect Dis 1999; 29(6): 1423-30.

[46] Grabar S, LeMoing V, Goujard C, et al. Clinical outcome of patients with HIV-1 infection according to immunologic and virologic response after 6 months of highly active antiretroviral therapy. Ann Intern Med 2000; 133(6): 401-10.

[47] Quinones-Mateu M, Arts E, Eds. HIV-1 fitness: Implications for drug resistance, disease progression, and global epidemic evolution. Los Alamos National Laboratory, Biology and Biophysics Group 2001.

[48] Gonzales L, Brindeiro RM, Aguiar RS, et al. Impact of nelfinavir resistance mutations on in vitro phenotype, fitness, and replication capacity of human immunodeficiency virus type 1 with subtype B and C proteases. Antimicrob Agents Chemother 2004; 48(9): 35525 .

(C) Kitchen et al.; Licensee Bentham Open.

This is an open access article licensed under the terms of the Creative Commons Attribution Non-Commercial License (http://creativecommons.org/licenses/by-nc /3.0/) which permits unrestricted, non-commercial use, distribution and reproduction in any medium, provided the work is properly cited. 